\title{
Le thallium dans les sols et les végétaux supérieurs. II. Le thallium dans les végétaux supérieurs
}

\author{
A Tremel, M Mench* \\ Unité d'agronomie, Inra, Centre de recherches Bordeaux-Aquitaine, BP 81, 33883 Villenave-d'Ornon cedex, France
}

(Reçu le 15 novembre 1996 ; accepté le 2 juin 1997)

\begin{abstract}
Summary - Thallium in plants. Owing to its possible toxic effect on plants or its accumulation in edible plant parts, high content of thallium in soils has to be considered as a hazard for the environment. The purpose of this paper is to review the most recent data regarding content, phytotoxicity, bioavailability, translocation and accumulation of the metal in plants leading to possible damage to the food chain. At the present time severe detrimental effects on crops or acute poisoning of a population induced by ingestion of thallium polluted plant parts have been scarcely observed. However, chronic toxicity resulting from continuous intake of thallium originating from plants can occur in some areas.
\end{abstract}

\section{thallium / toxicity / plant / food chain / trace element}

Résumé - En raison de sa toxicité pour la plupart des organismes, le thallium présent dans les sols représente dans quelques cas un risque pour l'environnement dans la mesure où il peut être à l'origine d'une phytotoxicité ou compromettre la qualité de la chaîne alimentaire en s'accumulant dans la partie consommable des plantes. Le but de cette note est de faire le point sur la connaissance actuelle concernant les concentrations, la phytodisponibilité, la phytotoxicité, le transfert et l'accumulation de ce métal dans les végétaux ainsi que les conséquences qui en dérivent en matière de qualité de la chaîne alimentaire. À ce jour très peu de cas de phytotoxicité sur cultures ou d'empoisonnements aigus provoqués par l'ingestion de végétaux pollués par du thallium ont été observés. En revanche, le risque d'une toxicité chronique résultant de la consommation continue d'aliments de nature végétale ne peut être exclu dans certaines zones.

thallium / toxicité / chaîne alimentaire / élément trace

\section{INTRODUCTION}

Le thallium ( $\mathrm{Tl})$, dont la concentration dans les organismes vivants est inférieure à $100 \mathrm{mg} \cdot \mathrm{kg}^{-1} \mathrm{MS}$ peut être qualifié d'élément trace (Adriano, 1986). À ce jour considéré comme non essentiel, il est toxique sous sa forme monovalente et trivalente pour des systèmes biologiques variés, levures, bactéries, plantes et animaux (Lester, 1987). Une dose de plus de $100 \mathrm{mg}$ (1,4 mg Tl. $\mathrm{kg}^{-1}$ de masse corporelle) entraîne une

* Correspondance et tirés à part

Tél : (33) 0556843042 ; fax : (33) 0556843054 ; courriel : mench@bordeaux.inra.fr 
toxicité aiguë chez un homme adulte, et une dose de 500 à $800 \mathrm{mg}$ est souvent mortelle. La dose létale 50 est de 8 à $12 \mathrm{mg} \mathrm{Tl} . \mathrm{kg}^{-1}$ chez l'homme (Mulkey et Oehme, 1993). Les principaux signes cliniques d'une thallotoxicose aiguë sont: gastroentérite, neuropathie périphérique d'étiologie inconnue, alopécie. Tous les organes sont touchés, aussi bien le tractus gastro-intestinal, le système nerveux, les yeux, la peau, les reins que le système reproducteur (Mulkey et Oehme, 1993). Une période de latence de quelques heures à $2 \mathrm{j}$ suit une exposition aiguë et de 6 semaines à 3 ou 4 mois une exposition subaiguë (Zartner-Nyilas et al, 1983). De faibles doses sur une longue période produisent les mêmes symptômes, plus atténués, et souvent apparaissent des symptômes non spécifiques. Un empoisonnement chronique peut produire de faibles effets ou des dégâts importants, selon la sévérité de l'exposition (Mulkey et Oehme, 1993). Les effets mutagènes et tératogènes du thallium sont controversés (Merian, 1991). Si l'empoisonnement est reconnu assez tôt, la guérison peut être totale, sinon les risques de séquelles au niveau des fonctions intellectuelles ou de décès sont grands (Mulkey et Oehme, 1993). Chez l'homme, le système nerveux périphérique peut conserver des séquelles pendant plusieurs mois ou années, et le système nerveux central des séquelles irréparables (troubles psychiques, délires, hallucinations, pertes de mémoires et problèmes oculaires) (Zartner-Nyilas et al, 1983).

Une voie d'exposition de l'homme au $\mathrm{Tl}$ est l'ingestion de produits végétaux renfermant ce dernier. La connaissance du cycle du métal dans le système sol-plante-aliments revêt donc une importance considérable. Le but de cette revue est de faire le point sur les acquis les plus récents dans ce domaine.

\section{CONCENTRATIONS EN THALLIUM RENCONTRÉES CHEZ LES VÉGÉTAUX SUPÉRIEURS}

\section{Concentrations habituelles}

La plupart des plantes récoltées sur des sols non pollués contiennent en moyenne de 0,02 à 0,05 mg Tl. $\mathrm{kg}^{-1}$ MS (Adriano, 1986; Jovic, 1993).

\section{Concentrations anormalement élevées}

En Macédoine, près de la frontière grecque, les sols de la région des mines d'Alsar contiennent
Tableau I. Concentrations en Tl des plantes d'Alsar, récoltées sur des sols contenant beaucoup de Tl d'origine géochimique, en mg. $\mathrm{kg}^{-1}$ de cendres (Zyka, 1970).

Espèce végétale

Concentration en $\mathrm{Tl}$

\begin{tabular}{lr}
\hline Galium sp Rubiaceae & 17000 \\
Campanula sp Campanulaceae & 5990 \\
Dianthus sp Caryophyllaceae & 5200 \\
$\begin{array}{l}\text { Linaria triphylla et } \\
\text { Onosma tauricum }\end{array}$ & $3000-3800$ \\
\hline
\end{tabular}

Tableau II. Concentrations en Tl des végétaux poussés à Guizhou, Chine, sur des sols contaminés par des déchets de mines de mercure et de charbon, en $\mathrm{mg} \cdot \mathrm{kg}^{-1}$ MS (Dai-Xing et Ding-Nan, 1985).

Espèce végétale

Concentration en $\mathrm{Tl}$

Chou chinois

$39,0-48,6$

Grains de riz et de céréales

$0,52-2,31$

beaucoup de Tl d'origine géochimique (orpiment, lorandite, vrbaite, barytine, calcédoine, réalgar, marcasite...). Les plantes spontanées de cette région ont des concentrations en $\mathrm{Tl}$ importantes (tableau I) et sont toxiques pour le bétail, dont la morphologie n'est pas affectée (Zyka, 1970). Les concentrations en $\mathrm{Tl}$ des sols ne sont pas précisées dans l'article.

De fortes concentrations ont été également rencontrées dans des végétaux cultivés dans des zones contaminées par du $\mathrm{Tl}$ d'origine anthropique (tableaux II et III).

\section{ABSORPTION DU THALLIUM PAR LE VÉGÉTAL}

\section{Prélèvement selon l'espèce végétale et répartition dans les organes de la plante}

Le prélèvement de $\mathrm{Tl}$ par les racines dépend des propriétés du sol, des conditions de culture et de l'espèce végétale (Lehn, 1986). Le facteur le plus important est l'espèce végétale (Umweltbundesamt Berlin, communication personnelle). Mais les données concernant le prélèvement selon l'espèce sont souvent contradictoires. Pour Sager (1986), les espèces accumulant le plus Tl sont le colza, le chou vert, la laitue, le chou rouge, le poi- 
Tableau III. Concentrations en $\mathrm{Tl}$ des végétaux poussés à Lengerich, Allemagne, sur des sols à différentes teneurs en $\mathrm{Tl}$ émis par une cimenterie, en mg. $\mathrm{kg}^{-1}$ de matière fraîche de légume prêt à être consommé (pelé, lavé, cuit...) (Crössmann, 1990).

\begin{tabular}{|c|c|c|c|}
\hline \multirow[b]{3}{*}{ Espèce végétale } & \multicolumn{3}{|c|}{$\begin{array}{c}\text { Concentration dans le sol } \\
\left.\text { (mg Tl. } \mathrm{kg}^{-1} \mathrm{MS}\right)\end{array}$} \\
\hline & 1,3 & 2,7 & 4,1 \\
\hline & \multicolumn{3}{|c|}{ Concentration dans la plante } \\
\hline Épinard & 0,40 & 0,45 & 0,74 \\
\hline Endive & 0,02 & 0,05 & 0,08 \\
\hline Carotte & 0,03 & 0,15 & 0,26 \\
\hline Salsifis & 0,24 & 1,12 & 1,54 \\
\hline Céleri (bulbe) & 0,03 & 0,09 & 0,11 \\
\hline Radis & 0,16 & 0,50 & 0,41 \\
\hline Poireau & 0,08 & 0,22 & 0,16 \\
\hline Pomme de terre & 0,07 & 0,23 & 0,47 \\
\hline Chou-rave & 0,37 & 1,72 & 4,89 \\
\hline Brocoli & 1,90 & 10,1 & 7,93 \\
\hline Chou vert & 2,91 & 33,4 & 57,0 \\
\hline
\end{tabular}

reau, la scarole et le trèfle. Pour Crössmann (communication personnelle), ce sont le colza, le brocoli et le chou vert qui ont les concentrations en $\mathrm{Tl}$ les plus élevées; l'endive, la laitue, la carotte, le chou rave, le chou de Bruxelles, le chou de Chine, le chou fleur, le chou rouge, l'oignon, le poireau, le haricot vert, le petit pois, la tomate, le concombre, le persil et la pomme de terre accumuleraient le moins, et le radis, la betterave rouge, le céleri, le navet, le salsifis, le chou frisé et l'épinard seraient des espèces intermédiaires. Cependant il existe une constante: le colza est l'espèce comestible chez laquelle on a trouvé les plus fortes concentrations (Schoer, 1984). Hoffmann et al (1982) notent en effet des concentrations atteignant $3300 \mathrm{mg} \mathrm{Tl} . \mathrm{kg}^{-1} \mathrm{MS}$ chez des plantes cultivées en pots, sur sol contenant du thallium originel. Crössmann (communication personnelle) a noté des coefficients de répartition sol-plante de 34 à 51 pour le colza. Le Tl d'origine géochimique est assimilable par cette espèce, puisque des graines de colza récoltées au champ sur un sol (leptosol rendzic) contenant $40 \mathrm{mg} \mathrm{Tl} . \mathrm{kg}^{-1} \mathrm{MS}$ ont une concentration de 9,7 mg Tl. $\mathrm{kg}^{-1} \mathrm{MS}$; de même, des parties aériennes de colza, récoltées sur un sol à $18 \mathrm{mg} \mathrm{Tl} . \mathrm{kg}^{-1}$ MS (luvisol stagnic), ont une concentration de $20 \mathrm{mg}$ Tl. $\mathrm{kg}^{-1} \mathrm{MS}$ (Tremel, 1996). Les raisons de l'accumulation de Tl par le colza sont complexes et font intervenir les parti- cularités physiologiques, morphologiques et anatomiques de cette espèce. Le colza est notamment connu pour mobiliser intensément les minéraux grâce à l'excrétion d'exsudats racinaires acides, qui augmenteraient la solubilité de $\mathrm{Tl}$ et d'autres éléments (eg, K) dans la rhizosphère (Crössmann, 1984; Hinsinger, 1990). D'autres facteurs liés au métabolisme du soufre, à la répartition du Tl dans les vacuoles et les parois, ainsi qu'à la grande surface racinaire sont à prendre en considération (Crössmann, communication personnelle). Le colza a la capacité de prélever rapidement le thallium par les racines (Tremel, 1996). Il prélève également le potassium avec facilité, ce qui conduit à un appauvrissement de la solution du sol en $\mathrm{K}$ et à la libération du $\mathrm{K}$ interfoliaire des illites (Hinsinger, 1990). Il est possible que le prélèvement de $\mathrm{Tl}$ par le colza dans des compartiments correspondant à la fraction non échangeable du sol soit lié à celui du K, la libération du $\mathrm{K}$ interfoliaire provoquant aussi celle de $\mathrm{Tl}$ interfoliaire (Tremel, 1996).

Les résultats contradictoires concernant l'accumulation de $\mathrm{Tl}$ par les plantes peuvent être expliqués par les différences de conditions de culture. De plus, pour chaque espèce, il est probable qu'il existe une variabilité de prélèvements selon la variété. Certains cas ont été décrits en Grèce où les plantes observées, bien que sans symptôme apparent, avaient des teneurs en Tl qui, pour d'autres situations, avaient entraîné des dégâts sur les végétaux (Schoer, 1984).

D'une façon générale, les plantes à cycles de végétation courts et systèmes racinaires peu développés prélèvent moins de $\mathrm{Tl}$.

La distribution du $\mathrm{Tl}$ dans les organes varie fortement avec l'espèce. Là encore, les résultats sont parfois contradictoires. Lehn (1986) remarque chez le colza une tendance à accumuler le $\mathrm{Tl}$ dans les racines, alors que Makridis et Amberger (1989b) et Tremel (1996) notent une accumulation dans les parties aériennes. Malgré tout, d'une façon générale, l'accumulation est plus marquée dans les organes végétatifs que reproducteurs (Umweltbundesamt, communication personnelle). En particulier les grains de blé et de maïs contiennent de très faibles concentrations en thallium, souvent inférieures à la limite de détection par l'ICP-MS, $4 \mu \mathrm{g}$ Tl. $\mathrm{kg}^{-1} \mathrm{MS}$ (Tremel, 1996). La teneur en Tl des racines semble être supérieure à celle des parties aériennes chez le blé d'hiver, l'orge de brasserie, le maïs, la moutarde blanche, la camomille et le cresson (Lehn, 1986). Cataldo et Wildung (1978) trouvent chez le soja des concentrations qui aug- 
mentent dans l'ordre suivant: gousses et graines $<$ tiges $<$ feuilles.

\section{Prélèvement selon les propriétés du sol}

Le transfert de $\mathrm{Tl}$ vers la plante se fait d'autant plus que le sol a un $\mathrm{pH}$ bas, des teneurs faibles en argiles et en matières organiques, et donc une capacité d'échange cationique peu importante. Ainsi le thallium des sols sableux est plus disponible (Umweltbundesamt Berlin, communication personnelle).

Le nitrate de $\mathrm{Tl}$ incorporé au sol est plus disponible que le $\mathrm{Tl}$ originel (Hoffmann et al, 1982). De même, $\mathrm{Tl}$ apporté sous forme de poussières de cimenterie dans des pots est plus disponible que le thallium d'origine géochimique, pour le colza, le haricot et le ray-grass (Makridis et Amberger, 1989a). Du colza, cultivé sur un sol pollué par une cimenterie, contenant 10 fois plus de $\mathrm{Tl}$ total que le sol témoin, accumule 37 fois plus de Tl que les plantes témoins (Lehn, 1986). Dans le sol non pollué, le Tl prélevé par la plante provient en majorité des fractions granulométriques fines et est fixé par adsorption (partie échangeable majoritaire), alors que dans le sol pollué le Tl absorbé ne provient pas d'une fraction granulométrique particulière, et est réparti de manière homogène dans toutes les phases du sol, avec cependant une accumulation préférentielle dans les fractions extraites par des réactifs forts (Lehn et Schoer, 1985; Lehn et Schoer, 1987).

L'utilisation de réactifs chimiques d'extraction pour évaluer la phytodisponibilité est peu probante car elle ne prend pas en compte le facteur plante. Dans des sols à forte concentration en Tl d'origine géochimique, la quantité présente dans les parties aériennes du maïs est corrélée à la fraction échangeable, alors que celle existant dans les parties aériennes de colza est corrélée à la fraction extraite par l'acide oxalique et l'oxalate d'ammonium à l'obscurité (Tremel, 1996). D'autre part, la phytodisponibilité de $\mathrm{Tl}$ est corrélée à la fraction soluble et échangeable pour les sols pollués par des retombées de cimenterie (Lengerich, Allemagne), alors qu'elle est corrélée au $\mathrm{Tl}$ total pour les sols dont $\mathrm{Tl}$ a une origine géochimique (Rasecke, 1991).

\section{Mécanismes de prélèvement par les racines}

Dans un leptosol rendzic contenant $30 \mathrm{mg}$ Tl. $\mathrm{kg}^{-1}$ MS, le flux de masse (mass flow) contribue pour
25-30\% au Tl prélevé par le maïs lors des 5 premières semaines de culture et pour $10-16 \%$ dans le cas du colza (Tremel, 1996).

La racine est une première barrière sélective à l'accumulation des ions de la solution du sol. La vitesse d'absorption de $\mathrm{Tl}$ en fonction de la concentration en $\mathrm{Tl}^{+}$de la solution montre deux phases, conformes à une modélisation de type Michaelis-Menten: la première courbe correspond aux concentrations de la solution de 0 à $1 \mu \mathrm{M} \mathrm{Tl}$ et la deuxième de 1 à $40 \mu \mathrm{M} \mathrm{Tl}$. Cette réponse non linéaire de l'absorption à la concentration de la solution de sol est interprétée par l'existence d'un contrôle métabolique (Cataldo et Wildung, 1978). Smith et al (1991) concluent à la même hypothèse en comparant le prélèvement et l'accumulation de $\mathrm{Tl}^{+}$et $\mathrm{Cd}^{2+}$ chez une plante aquatique, Lemna minor. $\mathrm{Cd}$ montre une grande affinité à se lier à des ligands, tandis que $\mathrm{Tl}$ se comporte comme un ion libre. La concentration de $\mathrm{Cd}$ dans la plante est linéaire en fonction de la concentration en $\mathrm{Cd}$ du milieu extérieur, alors que pour $\mathrm{Tl}$ il y a saturation d'un mécanisme d'absorption à partir d'une concentration dans le milieu extérieur de $4 \mu \mathrm{M}$. Ces résultats, associés à l'étude de l'influence d'inhibiteurs comme le vérapamil ou le vanadate, montrent que l'absorption de $\mathrm{Tl}$ est plus rapide et que la saturation des mécanismes d'absorption de ce métal est plus nette (Smith et al, 1991).

Des expériences sur des racines d'orge excisées, en solution nutritive contenant $5 \mathrm{mg} \mathrm{Tl} . \mathrm{kg}^{-1}$, ont montré que les mécanismes de prélèvement de $\mathrm{Tl}^{+}$et $\mathrm{Tl}^{3+}$ étaient différents. $\mathrm{Tl}^{+}$est plus rapidement absorbé que $\mathrm{Tl}^{3+}$; la plupart des ions $\mathrm{Tl}^{3+}$ prélevés par les racines sont éliminés par lavage à l'aide d'une solution d'un agent désorbant (l'EDTA étant le plus efficace), contrairement à $\mathrm{Tl}^{+}$. Le prélèvement de $\mathrm{Tl}^{+}$augmente avec la température, jusqu'à $30^{\circ} \mathrm{C}$, et diminue ensuite, alors que les effets sont moins évidents pour $\mathrm{Tl}^{3+}$; de même, le prélèvement de $\mathrm{Tl}^{+}$est fortement affecté par le vanadate et le DNP, et pas celui de $\mathrm{Tl}^{3+}$. Tout ceci montre que l'absorption de $\mathrm{Tl}^{+}$ correspond à un processus actif, où interviennent peut-être les phosphotransférases, $\mathrm{K}^{+}$étant en compétition pour les sites de prélèvement (Logan et $\mathrm{al}, 1983)$. Un ajout de $\mathrm{K}^{+}$inhibe de façon non compétitive l'absorption de $\mathrm{Tl}^{+}$(Cataldo et Wildung, 1978). La cinétique de prélèvement de $\mathrm{Tl}(\mathrm{I})$, étudiée sur des racines d'orges excisées, suit une courbe de Michaelis-Menten simple, dont la constante d'absorption $(\mathrm{Km})$ est supérieure à celle de la cinétique de prélèvement de $\mathrm{K}^{+}$. Le Tl est localisé par la suite dans le cytoplasme des cellules racinaires, alors que $\mathrm{Tl}^{3+}$ pourrait être 
prélevé par échange d'ion, et localisé dans l'espace extra cellulaire (Logan et al, 1983). Au niveau de la plante entière (maïs et tomate), il n'y a pas de différence de prélèvement entre Tl(I) et Tl(III). $\mathrm{Tl}(\mathrm{I})$ stimule les ATPsynthases isolées de maïs, et entraîne une extrusion de protons par les racines. Les plantes absorbent donc Tl(I) en l'état, Tl(III) étant réduit avant son prélèvement. Pour Logan (1985), $\mathrm{K}^{+}$et $\mathrm{Na}^{+}$inhibent le prélèvement de $\mathrm{Tl}^{+}$de façon compétitive. Des études concernant le prélèvement de $\mathrm{Tl}^{+}$par des racines excisées d'orge montrent que ce processus est influencé par les ions $\mathrm{K}^{+}($Logan et al, 1984): le prélèvement est inhibé par des taux croissants de $\mathrm{K}^{+}$dans la solution, et cet effet augmente avec le temps. Cependant des expérimentations au champ de fertilisation potassique croissante $(150$ et $300 \mathrm{~kg} \cdot \mathrm{ha}^{-1}$ ) sur épinard ne montrent pas d'influence de ce facteur sur l'absorption de Tl (Crössmann, communication personnelle).

Jones et al (1990) rapportent qu'une diminution de l'alimentation en $\mathrm{K}$ entraîne une augmentation du transport de $\mathrm{Tl}$, et un accroissement de la toxicité chez le maïs et la tomate. Les plantes carencées en $\mathrm{K}^{+}$en solution sont plus sujettes à des chutes de biomasse dues à l'accumulation de $\mathrm{Tl}$, bien que le transport de $\mathrm{Tl}$ des racines vers les parties aériennes ne change pas. Les concentrations en $\mathrm{K}$ dans la plante sont plus importantes quand la solution nutritive contient du $\mathrm{Tl}$, peutêtre parce que $\mathrm{Tl}^{+}$active le système d'ATPsynthase, permettant le prélèvement de $\mathrm{K}^{+}$ (Logan et al, 1984).

\section{SPÉCIATION DU THALLIUM DANS LA PLANTE}

La spéciation des métaux dans les végétaux conditionne leur toxicité pour l'organisme luimême et pour les animaux qui les ingèrent. Il semble que $\mathrm{Tl}^{+}$et $\mathrm{K}^{+}$suivent les mêmes voies de pénétration dans la racine ; en revanche, il n'en est pas de même pour le transport ultérieur dans la plante, car il n'y a pas de relation entre les concentrations en $\mathrm{Tl}$ et $\mathrm{K}$ dans les tissus de tomate (Logan et al, 1984), de chou, de navet, de raygrass, de maïs, d'aubergine, de blé, de laitue, de pomme de terre, de carotte, de haricot et d'épinard (Tremel, 1996).

Chez Lemma minor, environ $80 \%$ du thallium est sous forme soluble, et est associé à des composés de faible poids moléculaire, alors que $\mathrm{Cd}$ est associé à des polypeptides ou des protéines. Selon Smith et al (1991), Tl présent dans cette plante est surtout intracellulaire et à l'état d'ion libre. Au contraire, la moitié du $\mathrm{Cd}$ présent est extracellulaire, la majorité du $\mathrm{Cd}$ intracellulaire étant liée aux protéines du cytosol. Cela peut expliquer le caractère plus toxique de $\mathrm{Tl}$ que celui de $\mathrm{Cd}$, et la plus grande aptitude des plantes à accumuler $\mathrm{Cd}$. Cependant, ces résultats sont sans doute à nuancer si l'on tient compte du complexage de Cd par les phytochélatines (PC) et le stockage des complexes PC-Cd dans la vacuole. Le cadmium activerait plus l'enzyme PCsynthase que le thallium (Zenk, 1996).

Chez le colza contenant une faible concentration de $\mathrm{Tl}$, tout le métal est sous forme liée. La filtration sur gel du cytoplasme montre que le thallium est élué associé à un seul composé, de masse molaire $3800 \mathrm{~g} \cdot \mathrm{mol}^{-1}$, qui ne comporte pas d'acides aminés soufrés. Le cadmium se trouve sous trois formes chimiques différentes: une à haut poids moléculaire (plus de $80000 \mathrm{~g} \cdot \mathrm{mol}^{-1}$ ) et deux à faible poids (4400 g. $\left.\mathrm{mol}^{-1}\right)$ (Günther et Umland, 1988). Pour de faibles concentrations en Cd dans la plante, le métal est lié à la membrane, ou est transporté vers la vacuole, sous forme ionique libre ou chélaté par des phytochélatines (Wagner, 1993; Zenk, 1996).

Chez des plants de colza contaminés intentionnellement par du thallium, $95 \%$ du métal est sous forme ionique libre, et $0,3 \%$ sous forme de complexe peptidique. $\mathrm{Tl}$ sous forme trivalente ou diméthylée n'a pas pu être mis en évidence, $\mathrm{Tl}$ est donc vraisemblablement sous forme $\mathrm{Tl}^{+}$. La synthèse du peptide de liaison (phytochélatines?) n'augmente pas avec la concentration en $\mathrm{Tl}$ des tissus; l'hypothèse de l'intervention de ce peptide dans les mécanismes de tolérance du colza à Tl ne peut donc être proposée (Günther et Umland, 1988). Au contraire, les phytochélatines qui chélatent $\mathrm{Cd}$ sont induites quand la concentration en Cd cytoplasmique s'accroît (Steffens, 1990). Zenk (1996) rapporte que, sur la base des concentrations dans le milieu, l'effet de $\mathrm{Tl}^{3+}$ sur l'activité de l'enzyme PCsynthase est très inférieur à celui de $\mathrm{Cd}^{2+}$ et à ceux d'autres éléments comme As, $\mathrm{Pb}, \mathrm{Cu}$ et $\mathrm{Zn}$.

Au cours de la croissance de la plante, les métaux $\mathrm{Cd}$ et $\mathrm{Tl}$ se trouvent sous une forme de moins en moins extractible. Par exemple chez le colza, au stade $10 \mathrm{~cm}$ de haut, la majorité de $\mathrm{Tl}$ est sous forme soluble, alors que chez un colza à maturité (1,5 $\mathrm{m}$ de hauteur, en fleurs), environ $30 \%$ du métal est sous forme non soluble, c'està-dire lié aux grosses molécules de la paroi ou complexé par des composés lipophiles, alors que 
dans les mêmes conditions $80 \%$ du Cd est sous forme non soluble (Günther et Umland, 1988).

\section{PHYTOTOXICITÉ DU THALLIUM}

Des symptômes caractéristiques de la phytotoxicité du thallium n'ont jamais été observés en plein champ. Les résultats rapportés ci-dessous proviennent donc d'essais conduits en conditions contrôlées. La phytotoxicité du $\mathrm{Tl}$ se manifeste par un aspect chlorotique des feuilles, une ramification moindre des racines, une diminution de la biomasse racinaire et foliaire, puis la mort de la plante. Tl limite la photosynthèse, en particulier en altérant la synthèse de la chlorophylle, ce qui entraîne la décoloration de la feuille. Elle commence à la base de la nervure et progresse le long de cette dernière avant de s'étendre à toute la surface de la feuille (Schoer, 1984). L'intervention de $\mathrm{Tl}$ dans la régulation stomatique perturbe la transpiration. La germination est de même inhibée par Tl, et la nutrition minérale modifiée.

La sensibilité au $\mathrm{Tl}$ est variable selon l'espèce végétale (Allus et al, 1987), et le critère de toxicité retenu (paramètre physiologique mesuré, et la concentration du thallium dans le sol ou dans la plante). Par exemple en culture en pots, la croissance de la laitue diminue après un apport de $10 \mathrm{mg} \mathrm{Tl} . \mathrm{kg}^{-1} \mathrm{MS}$ dans le sol sous forme de nitrate, alors que la croissance du colza commence à être affectée à partir de $200 \mathrm{mg}$ Tl. $\mathrm{kg}^{-1}$ MS dans le sol (Makridis, 1987). Une diminution de rendement de $10 \%$ est observée chez le haricot pour $130 \mathrm{mg} \mathrm{Tl} . \mathrm{kg}^{-1} \mathrm{MS}$ dans la plante, et chez le colza pour $800 \mathrm{mg} \mathrm{Tl} . \mathrm{kg}^{-1}$ MS (Makridis et Amberger, 1989a). La concentration critique est la concentration en Tl mesurée dans la plante pour laquelle le rendement commence à diminuer significativement. La concentration critique pour du ray-grass de 7 semaines est de $0,71 \mathrm{mg} \mathrm{Tl} . \mathrm{kg}^{-1}$ MS pour les parties aériennes, et 2,1 pour les racines. La concentration létale est de $251,2 \mathrm{mg} \mathrm{Tl} . \mathrm{kg}^{-1} \mathrm{MS}$ pour les parties aériennes et $1990 \mathrm{mg} \mathrm{Tl} . \mathrm{kg}^{-1}$ MS pour les racines. La même expérience a montré que $\mathrm{Tl}$ était moins toxique que le $\mathrm{Cd}$ et le $\mathrm{Hg}$ si l'on se réfère à la concentration critique, mais que $\mathrm{Tl}$ était plus toxique que ces métaux sur la base de la concentration létale (Al Attar et al, 1988).

Selon Kaplan et al (1990), Tl exercerait un effet toxique en agissant sur le métabolisme cellulaire des racines, à l'origine d'une modification des équilibres des macro- et des micronutriments dans la plante. Chez le haricot et le soja, la concentration en $\mathrm{K}$ augmente, et celles de $\mathrm{Ca}$ et $\mathrm{Mg}$ diminuent dans les feuilles. Makridis et Amberger (1989a) observent que l'augmentation de Tl dans la solution nutritive entraîne une diminution des concentrations en $\mathrm{K}, \mathrm{Cu}, \mathrm{Zn}$ et $\mathrm{Fe}$ dans le haricot, et, chez le colza, une diminution des concentrations en $\mathrm{K}$ et $\mathrm{Cu}$ en même temps qu'une augmentation légère en $\mathrm{Ca}, \mathrm{Mg}, \mathrm{Mn}, \mathrm{Fe}$, et $\mathrm{Zn}$.

La mesure des échanges gazeux montre que $\mathrm{Tl}$ diminue la photosynthèse du tournesol de $50 \%$

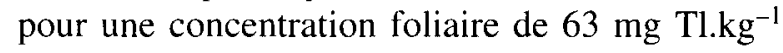
MS. Pour une même concentration en métal, la chute de photosynthèse est plus importante pour $\mathrm{Tl}$ que pour $\mathrm{Ni}, \mathrm{Cd}$, et $\mathrm{Pb}$. $\mathrm{Tl}$ est un inhibiteur plus efficace de l'ouverture des stomates que ces derniers métaux, peut-être en raison de la substitution de $\mathrm{K}$ par $\mathrm{Tl}$ dans les transporteurs de ce dernier (Bazzaz et al, 1974). Chez le maïs, l'ouverture des stomates est diminuée de $50 \%$ pour des concentrations dans les parties aériennes 10 fois plus faibles que pour $\mathrm{Ni}$ et $\mathrm{Cd}$. $\mathrm{Tl}$ est moins accumulé par les plantes que $\mathrm{Ni}, \mathrm{Cd}$ et $\mathrm{Pb}$, mais en revanche il diminue un peu plus la photosynthèse nette et la croissance. Le thallium inhibe plus la photosynthèse que la transpiration, ce qui signifie qu'il perturbe sans doute, outre l'ouverture des stomates, certaines étapes de l'assimilation du $\mathrm{CO}_{2}$ (Carlson et al, 1975; Tremel, 1996). L'absorption nette de $\mathrm{CO}_{2}$ semble être un marqueur précoce de toxicité due à $\mathrm{Tl}$ chez le maïs (tableau IV) (Tremel, 1996). Tl, comme Cd, Cr et $\mathrm{V}$ inhibe à de faibles concentrations la production

Tableau IV. Concentrations les plus élevées pour lesquelles on n'observe pas d'effet du Tl (NOEC) et concentrations les plus faibles pour lesquelles on observe un effet du Tl (LOEC) dans la quatrième feuille de maïs (en $\mathrm{mg} . \mathrm{kg}^{-1} \mathrm{MS}$ ) pour différents paramètres physiologiques ou de croissance (Tremel, 1996).

\begin{tabular}{llc}
\hline Marqueur & NOEC & LOEC \\
\hline $\begin{array}{l}\text { Absorption nette de } \mathrm{CO}_{2} \\
\begin{array}{l}\text { Résistance stomatique } \\
\quad \text { (face supérieure) }\end{array}\end{array}$ & 0,42 & 0,72 \\
$\begin{array}{l}\text { Intensité de la fluorescence } \\
\text { de la chlorophylle a }\end{array}$ & 33 & 41 \\
$\begin{array}{l}\text { Biomasse de F4 (MF et MS) } \\
\text { Densité de chlorophylle }\end{array}$ & 33 & 99 \\
$\begin{array}{l}\text { Teneur en eau } \\
\text { Résistance stomatique } \\
\text { (face inférieure) }\end{array}$ & 33 & 99 \\
\hline
\end{tabular}

$\mathrm{F} 4$ : quatrième feuille. 
d'oxygène, induite par la lumière, de l'algue Chorella saccharophila. Mais par rapport aux autres métaux, Tl modifie l'effet Kautsky de l'algue d'une manière spécifique: $\mathrm{Tl}$ inhibe la fonction réductrice du photosystème II, alors que $\mathrm{Cd}, \mathrm{Co}, \mathrm{Cu}, \mathrm{Ni}$ inhibent sa fonction oxydante. $\mathrm{La}$ sensibilité du système de transfert des électrons à l'accumulation du métal est plus faible pour Tl que pour $\mathrm{Pb}$ et $\mathrm{Cu}$, et plus forte que pour $\mathrm{Cd}$ (Wystrcil, 1984).

\section{CONSÉQUENCES POUR LA CHAINE ALIMENTAIRE DU THALLIUM PRÉSENT DANS LES VÉGÉTAUX. ASPECTS RÉGLEMENTAIRES}

La prise quotidienne de $\mathrm{Tl}$ acceptable selon l'EPA est de $0,53 \mu \mathrm{g} \mathrm{Tl} . \mathrm{kg}^{-1}$ de masse corporelle, soit $37,3 \mu \mathrm{g}$ pour un homme de $70 \mathrm{~kg}$ (Ewers, 1988). L'ingestion quotidienne moyenne aux États-Unis a été estimée à $5 \mu \mathrm{g}$, les plus fortes contributions provenant des champignons et des Brassicacées (Sherlock et Smart, 1986). Le prélèvement quotidien de $\mathrm{Tl}$ d'une personne habitant près d'une installation brûlant du charbon a été évalué à $10,5-12,6 \mu \mathrm{g}$, les plus fortes contributions provenant de l'ingestion des légumes (Zartner-Nyilas, 1987). Le chou constituerait le principal intrant de $\mathrm{Tl}$ dans la ration alimentaire française moyenne, puisqu'il représenterait $80 \%$ du $\mathrm{Tl}$ apporté par les légumes (Tremel, 1996).

En Allemagne, quand il y a dépassement des valeurs guides, il est recommandé de rechercher l'origine de la contamination, et d'essayer de supprimer la source de pollution. Pour les fruits et légumes, la valeur guide est de $0,1 \mathrm{mg} \mathrm{Tl} \cdot \mathrm{kg}^{-1}$ de matière fraîche (MF). Cette valeur a été calculée d'après des données statistiques $\left(95^{\circ}\right.$ percentile des concentrations couramment rencontrées), des considérations sur la santé humaine (ingestion quotidienne acceptable selon l'OMS) et de la ration alimentaire moyenne de la population. Cette valeur n'a pas pour le $\mathrm{Tl}$ de signification toxicologique, puisque seuls les fruits et légumes sont concernés (BGA, 1990). Pour les fourrages, il existe plusieurs valeurs, selon l'espèce animale censée les consommer. Le bœuf peut absorber des végétaux contenant jusqu'à $1 \mathrm{mg} \mathrm{Tl} . \mathrm{kg}^{-1}$ de fourrage à $88 \%$ de MS, le porc et le mouton jusqu'à $0,5 \mathrm{mg} \mathrm{Tl} \cdot \mathrm{kg}^{-1}$, les poules pondeuses $0,4 \mathrm{mg} \mathrm{Tl} \cdot \mathrm{kg}^{-1}$, et les poules à viande $0,2 \mathrm{mg} \mathrm{Tl} \cdot \mathrm{kg}^{-1}$. Ces valeurs ont été calculées en se basant sur des résultats expérimentaux et ont pour objet de protéger les consommateurs de viande. Elles correspondent à une accumulation significative de $\mathrm{Tl}$ dans les produits animaux (VDI 1992).

La transformation subie par les végétaux possédant une forte teneur en $\mathrm{Tl}$, avant leur ingestion, a une incidence sur le risque d'exposition des organismes consommateurs. Par exemple, le fait d'ensiler les fourrages entraine une perte de $\mathrm{Tl}$, par l'écoulement du jus de fermentation; de même, le thallium est entraîné dans l'eau de lavage et de cuisson des légumes: la cuisson diminue de $40 \%$ le $\mathrm{Tl}$ ingéré via les carottes, et de $67 \%$ celui issu du chou vert, en revanche la cuisson n'a pas d'effet sur la teneur en $\mathrm{Tl}$ du chou rave (Crössmann, communication personnelle).

Trois cas d'exposition importante au $\mathrm{Tl}$ de populations ont été recensés. L'exemple le plus connu est celui de Lengerich, en Allemagne, à proximité d'une cimenterie. En effet on a trouvé en 1979 dans ce secteur des fourrages à base de colza contenant $1095 \mathrm{mg} \mathrm{Tl} . \mathrm{kg}^{-1} \mathrm{MS}$, et ceux à base de maïs, $50 \mathrm{mg} \mathrm{Tl} . \mathrm{kg}^{-1} \mathrm{MS}$. Les concentrations en métal de certains organes de lapins, élevés à $500 \mathrm{~m}$ à l'ouest de l'usine, étaient très fortes (plus de $20 \mu \mathrm{g} \mathrm{Tl} . \mathrm{g}^{-1} \mathrm{MF}$ pour les reins, $3,9 \mu \mathrm{g} \mathrm{Tl} . \mathrm{g}^{-1} \mathrm{MF}$ pour le foie et $3,6 \mu \mathrm{g} \mathrm{Tl} . \mathrm{g}^{-1} \mathrm{MF}$ pour le muscle) (Landesanstalt für Immissionsschutz, 1980). La population locale a été contaminée, comme l'indiquent les concentrations plus élevées dans l'urine et les cheveux. Les cheveux renfermaient environ $10 \mathrm{ng} \mathrm{Tl.g}{ }^{-1} \mathrm{MS}$ en zone non contaminée alors qu'à Lengerich la moyenne sur 1163 personnes était de 20,3 ng Tl.g ${ }^{-1} \mathrm{MS}$, avec des valeurs pouvant atteindre $565 \mathrm{ng} \mathrm{Tl.g^{-1 }}$ MS. De même, les taux de Tl dans l'urine $\left(5,2 \mu \mathrm{g} . \mathrm{l}^{-1}\right)$ étaient environ 10 fois plus élevés que ceux trouvés dans une région non exposée $\left(0,4 \mu \mathrm{g} \mathrm{Tl} . \mathrm{L}^{-1}\right)$ (Schoer, 1984). L'augmentation de l'excrétion de $\mathrm{Tl}$ par les cheveux et l'urine était corrélée à des symptômes tels qu'insomnies, fatigue, faiblesse corporelle générale, nervosité, maux de tête, et à des troubles neurologiques comme paresthésie, ou des douleurs musculaires et articulaires (Schoer, 1984).

Un exemple moins connu, mais encore plus spectaculaire, est celui de la province de Ghuizhou, en Chine. De 1960 à 1977, 189 cas d'empoisonnement chronique au $\mathrm{Tl}$ ont été identifiés en liaison avec la consommation de choux chinois, cultivés sur des sols reposant sur des remblais de mines de $\mathrm{Hg}$ et de charbon. Les malades souffraient d'alopécies, de neuropathie périphérique, de désordres de la vue et gastrointestinaux. La plupart des patients traités n'ont pas eu de séquelles, sauf quatre d'entre eux, atteints de troubles visuels irréversibles. Au 
moment du diagnostic les concentrations de $\mathrm{Tl}$ dans l'urine étaient de 600 à $3000 \mu \mathrm{g} . \mathrm{L}^{-1}$, et de 6000 à 50000 ng. g $^{-1}$ MS dans les cheveux (DaiXing et Ding-Nan, 1985).

L'empoisonnement par le thallium d'une partie de la population de la ville de Chernotsy en Ukraine pendant plusieurs mois en 1988 et 1989 est également spectaculaire. Dans ces circonstances des centaines d'enfants ont souffert d'hallucinations et perdu leurs cheveux. La cause de la pollution n'a cependant pas été clairement élucidée (anonyme, 1989).

\section{CONCLUSION}

En France, les données actuellement disponibles en matière de concentrations en thallium des produits agricoles à destination alimentaire sont extrêmement rares. Dans ce domaine, les produits dérivant du colza et plus généralement des Brassicae seraient à examiner avec un soin particulier. On peut regretter par ailleurs que les écotoxicologues se soient assez peu intéressés aux indicateurs biologiques de pollution par ce métal, comme les mousses et lichens lorsque la dissémination s'effectue par voie atmosphérique (Jovic, 1993).

Au plan de la connaissance des mécanismes de transfert de $\mathrm{Tl}$ dans le système sol-plante, de nombreux points d'ombre subsistent. Par exemple l'explication de l'accumulation du métal dans l'espèce colza en est encore au stade des hypothèses. De même, sont encore mal connues la spéciation et la compartimentation de $\mathrm{Tl}$ dans les tissus du végétal, les processus de synergie ou d'antagonisme vis-à-vis d'autres éléments et notamment du potassium, les seuils de toxicité en fonction des espèces et des variétés. Ce type de problématique est certes commun à la plupart des éléments traces impliqués dans le système sol-plante ; cependant, la grande toxicité spécifique de $\mathrm{Tl}$ ( $37 \mu \mathrm{g}$ représente le maximum acceptable dans la ration quotidienne, à comparer à l'intervalle $57-71 \mu \mathrm{g}$ pour le cadmium) et sa relative abondance dans les sols (médiane des concentrations analogue à celle de $\mathrm{Cd}$ [Tremel et al, 1997]), sont des raisons qui militent pour une amélioration significative de la connaissance dans ce domaine.

\section{REMERCIEMENTS}

Ce travail a été réalisé grâce au soutien financier de l'Ademe (Agence de l'environnement et de la maîtrise de l'énergie), département Agriculture et Alimen- tation, Angers (contrat $n^{\circ} 4.75 .0016$, responsable I Feix). Les auteurs remercient $C$ Juste pour ses suggestions.

\section{RÉFÉRENCES}

Adriano DC (1986) Trace Elements in the Terrestrial Environment. Springer-Verlag, New-York

Al-Attar AF, Martin MH, Nickless G (1988) Uptake and toxicity of cadmium, mercury and thallium to Lolium perenne seedlings. Chemosph 17, 12191225

Allus MA, Martin MH, Nickless G (1987) Comparative toxicity of thallium to two plant species. Chemosph 16, 929-932

Anonyme (1989) Hair-raising. New Sci 28 January, 1649,28

Bazzaz FA, Carlson RW, Rolfe GL (1974) The effect of heavy metals on plants: Part I : inhibition of gas exchange in sunflower by $\mathrm{Pb}, \mathrm{Cd}, \mathrm{Ni}$ and $\mathrm{Tl}$. Environ Pollut 7, 241-246

BGA (Bundesgesundheitsamt) (1990) Richtwerte für Schadstoffe in Lebensmitteln. Bundesgesundheitsblatt 12, 224-226

Carlson RW, Bazzaz FA, Rolfe GL (1975) The Effect of heavy metals on plants, II. Net Photosynthesis and transpiration of whole corn and sunflower plants treated with $\mathrm{Pb}, \mathrm{Cd}, \mathrm{Ni}$, and $\mathrm{Tl}$. Environ Res $10,113-120$

Cataldo DA, Wildung RE (1978) Soil and plant factors influencing the accumulation of heavy metals by plants. Environ Health Perspect 27, 149-159

Crössmann G (1984) Thallium-eine neue Umweltkontaminante? Angew Bot 58, 3-10

Crössmann G (1990) Zur Mobilität und Akkumulation ausgewählter anorganisher und organisher Schadstoffe bei Pflanzen und Nutztieren. VDI Berichte 837, 799-813

Dai-Xing Z, Ding-Nan L (1985) Chronic thallium poisoning in a rural area of Guizhou Province, China. $J$ Environ Health 48, 14-18

Ewers U (1988) Environmental exposure to thallium. Sci Total Envir 71, 285-292

Günther K, Umland F (1988) Speziesanalytik von Cadmium and Thallium in nativen Rapspflanzen (Brassica napus). Fresen $Z$ Anal Chem 331, 302309

Hinsinger P (1990) Action des racines sur la libération du potassium et l'altération de minéraux silicatés, incidences agronomiques. Thèse de doctorat, Ensam, Montpellier

Hoffman GG, Schweiger P, Scholl W (1982) Aufnahme von Thallium durch landwirtschaftliche und gärtnerische Nutzpflanzen. Landwirtsch Forschung 35, 45-54 
Jones KC, Lepp NW, Obbard JP (1990) Other metals and metalloids. In : Heavy Metals in Soils (Alloway BJ, ed), John Wiley and Sons Inc, New York, 26

Jovic V (1993) Thallium in rocks, soils and plants: past progress and future needs. $N \mathrm{~J} b$ Miner $A b h$ $166,43-52$

Kaplan DI, Adriano DC, Sajwan KS (1990) Thallium toxicity in bean. J Environ Qual 19, 359-365

Landesanstalt für Immissionsschutz (1980) Umweltbelastung durch Thallium, Untersuchungen in der Umgebung der Dyckerhoff-Zementwerke $A G$ in Lengerich sowie anderer Thalliumemittenten im Lande $N W$. Minister für Arbeit, Gesundheit und Soziales und Minister für Ernährung, Landwirschaft und Forsten des Landes NW, Düsseldorf

Lehn H (1986) Aufnahme und Verteilung von Thallium, Cadmium, Blei, und Zink in ausgewählten Nutzpflanzen. Dissertation, Ruprecht-KarlsUniversität, Heidelberg

Lehn H, Schoer J (1985) Thallium transfer from soils to plants: relations between chemical forms and plant uptake. In: Heavy Metals in the Environment (Lekkas TD, ed), Inter Conf, Athens, vol II, CEP Consultants Ltd, Edinburgh, 286-288

Lehn H, Schoer J (1987) Thallium-Transfer von Böden in Pflanzen. Korrelation zwischen chemischer Form und Pflanzenaufnahme. Plant Soil 97, 253-265

Lester JN (1987) Heavy Metals in Wastewater and Sludge Treatment Process. Vol 1 : Sources, Analysis and Legislation. CRC Press Inc, BocaRaton

Logan PG (1985) Thallium uptake and transport in higher plants. PhD thesis, Department of Biology, Liverpool Polytechnic, Liverpool

Logan PG, Lepp NW, Phipps DA (1983) Thallium uptake by higher plants. 4th Conference on Heavy Metals in the Environment, 642-645

Logan PG, Lepp NW, Phipps DA (1984) Potassium nutrition and thallium toxicity in higher plants. VIth Intern Coll for the optimization of plant nutrition, Montpellier, Proc 2, 345-350

Makridis H (1987) Wirkung des Thallium in Zementofenstäuben auf Wachstum und Thallium Aufnahme mehrerer Kulturpflanzen zur Ermittlung von Thallium-Grenzwerten in Pflanzen und Böden. Dissertation, Fakultät für Landwirtschaft und Gartenbau der technischen Universität, Munich

Makridis H, Amberger A (1989a) Wirkung steigender Thallium-Konzentrationen auf Stoffproduktion und Aufnahme von Thallium und anderen Mineralstoffen durch Buschbonen und Grünraps in Nährlösungsversuchen. Landwirtsch Forschung 42, 333-343

Makridis H, Amberger A (1989b) Thallium Aufnahme aus Zementofenstäuben in Gefässversuchen mit Grünraps, Buschbohnen, und Weidelgras. Landwirtsch Forschung 42, 324-332
Merian E (1991) Metals and their Compounds in the Environment, Occurrence, Analysis and Biological Relevance. $\mathrm{VCH}$, Weinheim

Mulkey JP, Oehme FW (1993) A review of thallium toxicity. Vet Human Toxicol 35, 445-453

Rasecke S (1991) Gehalte und Bindungsformen von Tl in geogen und anthropogen belasteten Böden des Sauerlandes und Teutoburger Waldes. Diplomarbeit, Westfälische Wilhelms-Universität Münster, Institut für Geographie

Sager M (1986) Spurenanalytik des Thalliums. Georg Thieme Verlag, Stuttgart

Schoer J (1984) Thallium. In: The Handbook of Environmental Chemistry (Hutzinger O, ed), Vol 3, part C: Anthropogenic compounds. Springer Verlag, Berlin, 143-214

Sherlock JC, Smart GA (1986) Thallium in foods and the diet. Food Addit Contam 3-4, 363-370

Smith S, Kwan KHM, Mannings S (1991) Bioavailability and accumulated forms of trace metals in plants. In : Metal Compounds in Environment and Life (Merian E, Haerdi W, etd), Science and Technology Letters, Northwood, 127-135

Steffens (1990) The heavy metal-binding peptides of plants. Annu Rev Plant Physiol Plant Mol Biol 41, 55-575

Tremel A (1996) Transfert du thallium du sol vers la plante: caractérisation du risque dans les agrosystèmes. Thèse de doctorat, INPL Nancy

Tremel A, Masson P, Sterckeman T, Baize D, Mench M (1997) Thallium in French agrosystems. I. Thallium contents in arable soils. Environ Pollut 95 , 293-302

VDI (Verein Deutscher Ingenieure) (1992) Maximale Immissions-Werte für Thallium zum Schutz des landwirtschaftlichen Nutztiere. VDI-Richtlinien 2310 , Blatt 29

Wagner (1993) Accumulation of cadmium in crop plants and its consequences to human health. Advan Agron 51, 173-205

Wystrcil HG (1984) Untersuchungen über die Wirkung von Thallium auf die Primärprozesse der Photosynthese grüner Pflanzen. Dissertation, Institut für Landeskultur und Pflanzenökologie der Universität Hohenheim, Hohenheim

Zartner-Nyilas G (1987) Thallium. Bund-LänderArbeitskreis Umweltchemikalien, BadenWurttemberg, Karlsruhe

Zartner-Nyilas G, Valentin H, Schaller KH, Schiele R (1983) Thallium-ökologische, umwelt-medizinische und industrielle Bedeutung. Agras- und Umweltforschung in Baden-Württemberg, Band 3, Verlag Eugen Ulmer, Stuttgart

Zenk MH (1996) Heavy metal detoxification in higher plants - a review. Gene 179, 21-30

Zyka V (1970) Thallium in plants from Alsar Sb. Geol Ved Technol Geochem 10, 91-95 